\title{
FRANÇOIS POULLAIN DE LA BARRE: FILÓSOFO FEMINISTA Y CARTESIANO SUI GENERIS
}

\section{FRANÇOIS POULLAIN DE LA BARRE: FEMINIST PHILOSOPHER AND SUI GENERIS CARTESIAN}

\author{
LUZ STELLA LEÓN*
}

UNED

RESUMEN: Este artículo trata de introducir brevemente la vida y obra de François Poullain de la Barre, filósofo feminista y cartesiano sui generis del siglo XVII, dando a conocer la deriva social de los principios cartesianos.

PALABRAS ClavE: Poullain de la Barre, Modernidad, Ilustración, racionalismo, filosofía cartesiana, feminismo, igualdad.

ABSTRACT: This article briefly introduces the life and work of François Poullain, feminist philosopher and $17^{\text {th }}$ century sui generis Cartesian, announcing the social drift of Cartesian principles.

KEYwORDS: Modernity, Enlightenment, rationalism, Cartesian philosophy, feminism, equality.

\section{Introducción}

François Poullain de la Barre nació en París en julio de 1647 y murió en Suiza en mayo de 1725 . Fue un filósofo y pensador feminista del siglo XVII que

* Este artículo forma parte de mi investigación para la tesis doctoral: Feminismo y Filosofía social en François Poullain de la Barre. Todas las obras que se citan de diferentes idiomas son traducción propia, de modo contrario se especificará. 
vivió en el contexto de la Francia de Luis XIV y estuvo inmerso en lo que Paul Hazard denominara la «crisis de la conciencia europea» ${ }^{1}$.

En este artículo pretendemos introducir brevemente su «aventura filosófica» ${ }^{2}$, poniendo de relieve que sus elecciones filosóficas y sus elecciones existenciales, coinciden marcando su itinerario vital e intelectual.

En España la introducción del pensamiento de Poullain viene de la mano de Celia Amorós, que lo señala como un precursor pre-ilustrado del feminismo, afirmando que nuestro autor es producto del cartesianismo y del movimiento preciosista, y que su pensamiento representa «un momento particularmente lúcido y pregnante de la autoconciencia crítica de la especie en una fase muy significativa de su evolución social y cultural» ${ }^{3}$.

\section{Breve apunte biográfico}

La primera noticia biográfica de François Poullain de la Barre ${ }^{4}$ sobre la que tenemos conocimiento es de El gran diccionario editado en $1733^{5}$ de Moreri, quien, al parecer, conocía bien a nuestro autor.

${ }^{1}$ Hazard situó los orígenes de la Ilustración en el período que comprende aproximadamente entre 1680 y 1715, época de gran influencia del pensamiento cartesiano, y donde además empezaban a despuntar grandes nombres como Spinoza, Malebranche, Fontenelle, Locke, Leibniz, Bossuet, Fènelon, Bayle, entre otros. Cfr. HAZARD, (1988). La crisis de la conciencia europea, versión española de Julián Marías, Madrid, Alianza Universidad.

${ }^{2}$ Tal es título de la obra filosófica de Madeleine Alcover, editada en 1981. Es un libro dedicado a la figura y la obra de Poullain de la Barre, articulando su pensamiento con otros temas importantes del siglo XVII: XVIIème siècle como la religión y el protestantismo, la lengua latina y la lengua francesa, el individuo y la sociedad civil. Cfr. ALCOVER, (1981): Poullain de la Barre: une aventure philosophique, Papers on French Seventeenth Century Literature, Paris-Seattle-Tuebingen.

${ }^{3}$ AMOROS, (1995), Tiempo de feminismo. Sobre feminismo, proyecto ilustrado y postmodernidad, Madrid, Cátedra, Colección Feminismos, p. 121. La Educación de las Damas fue la primera obra de Poullain traducida al español por Ana Amorós para la colección feminismos de Cátedra.

${ }^{4}$ Desafortunadamente no contamos con su imagen ni con toda la documentación necesaria para desarrollar una biografía exacta de su vida, pero hemos rastreado huellas que nos permiten marcar una trayectoria intelectual y vital más o menos clara, y apoyándonos en las pesquisas de Stock entre otros, podemos reconstruir y elaborar hipótesis plausibles. En la propia composición del nombre de nuestro autor ya encontramos variaciones en cuanto a su ortografía y su composición, además en los textos tanto históricos como oficiales en los que no encontramos acuerdo ni concordancia; por ejemplo en una de sus firmas originales, el testamento en Genève firma como 
Poulain perteneció a una familia burguesa, su padre le destinó a la carrera eclesiástica y desde la edad de nueve años estuvo formándose para ella. Obtuvo el grado de Maestría a los 16 años, y tres años más tarde, en 1666, el grado de Bachiller de teología en la Sorbona, continuando su trayectoria con los estudios de doctorado en Teología — que nunca llegó a terminar por decisión propia—. Llevó una existencia poco corriente, aunque su familia le destinara al sacerdocio, desarrolló muy pronto un espíritu crítico e inconforme que le llevó a enfrentarse con el escolasticismo imperante y a mantener un continúo enfrentamiento con las jerarquías.

Desencantado del escolasticismo frecuentó l'Académie des Orateurs y asistió a las conferencias de un filósofo cartesiano, probablemente Jacques Rohault, y rápidamente se convirtió a la nueva filosofía ${ }^{6}$. Conocer las obras de Descartes, y recibir su influencia inmediata, fue para él como una verdadera "conversión» ${ }^{7}$;

François Poullain de la Barre, en otro firma como Poullain, sólo F. p. de la Barre, etc. Cfr. Alcover, op. cit pp. 9-11.

Las referencias biográficas más concretas las podemos rastrear en las obras de Stock, (1961) «Poullain de la Barre, a seventeenth century feminist», These dactylographiée de Doctorat, Columbia University, New York, pp. 1-90 et Appendices, pp. 223-232; Alcover, (1981) op., cit., p. 9-36; Magné, (1964) Le Féminisme de Poullain de la Barre, origine et signification, thèse dactylographiée pour le doctorat de 3ème cycle. Université de Toulouse; Stuurman, (2004), François Poullain de la Barre and the Invention of Modern Equality, Cambridge Mass \& London, Harvard University Press, pp. 24-50.

${ }^{5}$ El gran diccionario histórico de Moreri, edité à Bâle en 1733, vol. 6 pp. 1101. En el sexto volumen de la obra figura en cabeza el artículo Barre, pero parece que no fue considerado luego por los editores, que aunque lo recibieron tal cual, fue borrado en posteriores ediciones del mismo. Cfr. Alcover, p. 9 y Stock (1961), op. cit., pp. 11-12.

${ }^{6}$ Nuestro autor relata en l'Éducation: «Je me mis un jour, dit-il en parlant de la Sorbonne, je me mis un jour à faire réflexion sur ce que j’y avais appris. Je fus assez étonné de trouver que j’avais perdu ma peine, et que je n'étais habile qu'en parchemin et dans mes lettres de capacité... Je remarquais que tout ce queje savais n'était d'aucun usagedans le monde que pour faire fortune par une certaine voie où je ne voulais pas entrer. Je voyais que les honnêtes gens ne pouvaient souffrir notre manière de raisonner: que même je ne la pouvais guère employer qu'en latin; que l'on me démontait entièrement lorsque l'on m'obligeait de m'expliquer intelligiblement et de ne me point servir de certains mots et de certaines phrases queje prétendais être consacrés...; enfin qu'après avoir étudié depuis neuf ans jusqu'à vingt, avec beaucoup d'application et de succès pour un écolier, je n'étais guère plus avancé que si je n'eusse jamais rien fait, et qu'il me fallait recommencer tout de nouveau, selon l'avis de quelques personnes avec qui je m'entretenais» Poullain escribe sobre su "conversión» al cartesianismo en su tratado De l'éducation des dames pour la conduite de l'esprit dans les sciences et dans les moeurs, editado en París en 1674, pp. 87-88 - 332-336.

${ }^{7}$ Cfr. Grappin, (1913), «Notes sur un féministe oublié: le cartésien Poullain de la Barre,» Revue d'Histoire Littéraire de la France, 20, France, pp. 852-867 
encontró por fin el medio de encauzar sus inclinaciones personales e intelectuales, manteniendo la coherencia intelectual hasta su muerte.

Su formación cartesiana se desarrolló en una época en la que la escena intelectual francesa estaba dominada por la querelle entre los Antiguos y los Modernos, por la primera generación del cartesianismo y por el movimiento preciosista, respectivamente —el paso des ruelles del siglo XVII a los salones del XVIII-. Era una época de crisis y de cambio que bullía en las calles, en los salones, y en los círculos de oradores parisinos y en la que él, al parecer en sus primero años, pudo participar activamente. Por su formación y medio social seguramente participó en los debates que eran reflejo de la transición intelectual de su tiempo. En los círculos universitarios habían calado profundamente las disputas jansenistas que querían una reforma interior del pietismo y el agustinismo, en contra de los jesuitas que buscaban la reforma católica y estaban más próximos al poder. Pronto entró en contacto con las corrientes cartesianas y gasendistas que, aunque excluidas de la universidad, eran ampliamente leídas y discutidas en las calles y los salones parisinos de la época. También leyó los escritos de Arnault que en su tiempo despertaron grandes polémicas teológicas entre algunos de los estudiantes de la Sorbona.

Se convierte en un libre pensador radical que interpreta, de inmediato, aquellas doctrinas que agitan el espíritu de sus contemporáneos. Todo ello transformado bajo la influencia de un cartesianismo, audazmente interpretado.

Durante esos años Poulain se limita a leer la Escritura Sagrada, sobre todo el Nuevo Testamento y los Tratados de Descartes. Durante los siguientes diez años — de los veinte a los treinta años—, se dedica a escribir y publicar sus obras feministas de inspiración netamente cartesiana; al parecer, y según reseña Hernri Grappin, intentó ganarse la vida como autor de sus libros, pero no pudo ser, viéndose obligado a ganarse el pan a duras penas, a pesar de que sus obras no pasaron desapercibidas. En todo caso, ello no fue suficiente para asegurarse su existencia, por lo que tuvo que resignarse, finalmente, con consagrarse como sacerdote a los treinta años, en 1680. Fue nombrado párroco de la Flamangrie, en la diócesis de Laon, sobre la frontera de Picardie. Seguramente fue perseguido por sus ideas y desterrado de París, siendo destinado a parroquias pobres y lejanas. La desafortunada experiencia le lleva, a sus cuarenta y un años de edad, a abandonar su carrera eclesiástica en 1668 (sin reconocimiento oficial) y abrazar el protestan- 
tismo. Hacía falta un cierto coraje para adscribirse al protestantismo públicamente.

Finalmente Poulain no tuvo fortuna y sufrió las consecuencias prácticas de su apostasía. Obedeciendo fielmente a lo que le dictaba su conciencia, inmediatamente después de su conversión, regresa a París esperando poder reunir los medios de subsistencia y retirarse. Pero su apostasía le valió, no sólo el repudio definitivo de su familia, sino además, el ser perseguido y posteriormente tener que huir a Ginebra después de la revocación del edicto de Nantes en 1685.

Sobre este período de su vida tampoco tenemos muchos datos, excepto que llevó una existencia precaria en los primeros años dando clases de francés y de filosofía a jóvenes extranjeros; que se casó en 1690 y tuvo un hijo y una hija, y que publicó un último libro. El gobierno ginebrino otorga a Poullain, quizás tardíamente, una cátedra de secundaria en el collège de Genève. Un poco más tarde, en 1716, los señores del Petit-Conseil le concedieron el título de burgués, que nuestro autor pagó para él y su hijo. En mayo de 1723, a la edad de 76 años muere Poulain de la Barre. Por su testamento sabemos que se mantuvo firme en sus convicciones, identificándose hasta su muerte con el quehacer filosófico ${ }^{8}$ y, por su biografía, podemos entrever, como afirma Alcover, que llevó «una existencia poco ordinaria»?

En su trayectoria Poullain privilegió tres temas: la lengua francesa, es decir la apuesta por la Modernidad, la igualdad de los sexos con el fondo de la idea de la igualdad para todos los hombres y la necesidad del libre examen individual en materia de religión. Tres elecciones teóricas que son también elecciones prácticas. La filosofía cartesiana, y especialmente el Método, marcaron decisivamente la vida y obra de nuestro autor ${ }^{10}$.

${ }^{8}$ Ver Magnè, (1964), op. cit., Apéndice II, p. 407.

${ }^{9}$ Cfr. Alcover, (1981) op. cit., p. 9.

${ }^{10}$ Es importante subrayar que en su pensamiento influyeron, en la misma medida, tanto el cartesianismo y otras corrientes filosóficas como el movimiento de las preciosas y la querelle de las femmes, como contexto social; además de la querelle des Anciens et des Modernes ou querelle des Classiques et des Modernes. Las aristócratas cultas apoyaban el desarrollo de la lengua francesa, cuestionaban la autoridad marital, pedían el acceso al mundo intelectual, a las Academias, etc. Se convirtieron en «mediadoras» de ese nuevo modelo de sociabilidad de los salones literarios donde hombres y algunas mujeres_-nobles — discutían de todos los tópicos en igualdad, donde sólo los 


\section{La publicación de sus textos*}

Nuestro autor produjo algunos de los textos más radicales que se imprimieron en el siglo XVII. Sus obras corrieron diferente suerte, ya que algunas fueron reimpresas y traducidas, en ocasiones plagiadas o asignadas a otros autores, etc. Publica tres libros sobre las mujeres entre 1673 y 1675. De l'Egalité des deux sexes; De l'Education des Dames, y De l'Excellence des Hommes, contre l'égalité des Sexes.

En 1672 publica una obra en favor de la lengua francesa y en detrimento del Latín: Les Rapports de la langue Latine avec la Françoise, pour traduire élégamment et sans peine. - Recordemos que la primera obra escrita en francés es el Discurso del Método de Descartes publicada en 1637- y ya en 1691 Essai des remarques particulières sur la langue Françoise pour la ville de Genève. Su última publicación conocida fue La Doctrine des protestants sur la liberté de lire l'Ecriture Sainte etc.; editado en 1720 en la cual nuestro racionalista discurre sobre la cuestión de la libertad religiosa tema que fue central en la vida de Poullain ${ }^{11}$.

argumentos eran válidos. Se puede afirmar que desde finales del siglo XVI la «cuestión femenina» era un tema importante y polémico, tratado desde la literatura galante hasta la literatura abiertamente misógina, ridiculizado en Molière y muchos otros detractores. La querelle de femme tiene un largo recorrido y una amplia problemática que abarca por ejemplo: la lucha por ser escuchadas, educadas, por acceder al saber de las academias, etc. Durante siglos se discutió sobre el lugar que correspondía a cada sexo. En el siglo XVII corrieron ríos de tinta a favor y en contra de la querelle, por ejemplo sobre la forma y la necesidad de educar a las niñas. Tanto partidarios como detractores de les femmes escribieron gran cantidad de obras al respecto, pero sólo nuestro autor se ocupó del tema haciendo de las mujeres su «sujeto epistemológico». Cfr. Ascoli Georges (1912) «Essai Sur l'histoire des idées féministes en France du XVI e siècle a la Révolution» Revue de synthèse historique, Paris, Librairie Léopold Cerf. Siècles XVII y XVII 1906/08 (Tome 13) p. 25 -57 y 99-106 ; Reynier, Gustave (1929) La fermme au XVIIe siècle: ses ennemis et ses défenseurs, Paris, éditions J. Tallandier; Blanco C. Oliva, «La 'Querelle feministe' en el siglo XVII. La ambigüedad de un término: del elogio al vituperio", en C. Amorós (coord.), Actas del Seminario permanente "Feminismo e Ilustración", 1989-1992, UCM e Instituto de Investigaciones Feministas, Madrid, 1992. pp. 7383. Véase también Ginervra Conti d'Odorisio, Poulain de la Barre e la teoría de l'uguaglianza, Milán, Edizioni Unicopli, 1996. Entre otros.

* Para facilitar las citas en adelante se abreviaran las obras y se citarán como: L'Égalité, edición de 1673; L'Education edición de 1674 y L’Excellence edición de 1675.

${ }^{11} \mathrm{Al}$ respecto Stuurman señala que en nuestro autor hay una tensión irresuelta entre la idea de libertad y la noción de verdad religiosa y autoridad religiosa, una vez más el filósofo se adelanta a la Ilustración, Cfr. Stuurman, (2004), op., cit., p. 51. 
Ahora bien, para continuar con nuestra argumentación debemos detenernos en sus obras feministas: su primera obra en favor de la igualdad de los sexos no es aislada ni accidental, pues expresa el interés intelectual de Poullain por el feminismo como punto de partida en su proyecto racionalizador.

En 1673 escribió De l'égalité des deux sexes, discours physique et moral où l'on voit l'importance de se défaire des préjugez, editada en París, chez Jean du Puis 1673, 1676, 1679, 1690 y en 1692, respectivamente. Como señala Amorós, nuestro autor cartesiano quiere empezar por: «derivar en favor de los derechos de las mujeres, las implicaciones de la crítica cartesiana del prejuicio, la tradición y el argumento de autoridad, así como del dualismo mente-cuerpo» ${ }^{12}$. Este libro tiene una gran importancia filosófica, porque es la primera vez que las mujeres se convierten en "sujetos epistemológicos» de referencia para tratar de desvelar el mayor de los prejuicios, la desigualdad sexual, para desde ahí pensar los enigmas filosóficos de su época.

El segundo tratado De l'éducation des dames pour la conduite de l'esprit dans les sciences et dans les mours, es editado en París en 1674 y en Amsterdam en 1679, es presentado en forma de diálogo y tiene una gran trascendencia. Si bien la educación de las damas era un asunto muy discutido en la época, la novedad está en que nuestro autor propone un método educativo, una propuesta cartesiana concreta que sienta las bases para un programa educativo igualitario y universal para ambos sexos. En ésta y en sus demás publicaciones no cesa de reivindicar que el cerebro no tiene sexo, que el conocimiento y la educación son accesibles a todos por igual, y por tanto, que todos podemos participar en la vida pública ${ }^{13}$. La cuestión de su educación ilustra perfectamente los límites de la actitud liberal de algunos intelectuales de la época: la aparición de una nueva clase de mujeres ociosas, alimentadas sobre todo por la burguesía, había creado un problema social, por tanto, era necesario "procurar a estas damas algo de que ocuparse, tenían pues que permitirles abrir ciertos libros». Pero Poullain, a diferencia de éstos, no quiere educar a las mujeres para mantenerlas ocupadas, sino para que ocuparan su lugar en la sociedad y para que ejercieran su libertad.

${ }^{12}$ Cfr. Amorós, (1995), op. cit., p. 110 y ss. Subrayados nuestros.

${ }^{13}$ Cfr. Magnè, (1972), "Introduction à la réédition " De l'Egalité des deux sexes a paraître aux éditions Privait, collections «Résurgences». Veáse además del mismo autor «Education des femmes et feminisme chez Poullain de la Barre (1674-1723)», Le XVII siècle et l'education, colloque de Marsella, 1971, en Supplément au no 88, Revue Marseille. 
Es al final de esta obra que nuestro autor se declarará abiertamente cartesiano ${ }^{14}$. Pero no es un seguidor dogmático, simplemente considera que es el mejor método que ha encontrado hasta el momento: «Yo no pretendo apuntar aquí que Descartes sea infalible, que todo lo que ha avanzado sea verdadero y sin dificultad, y que sobreviva ciegamente y que los otros no puedan encontrar mejores caminos que los que él encontró. Solamente les digo que es uno de los filósofos más razonables de los que he leído» ${ }^{15}$. Por esto recomienda la lectura de Gassendi, de Coreau de La Chambre y de Lesclashe, los tres opuestos a Descartes.

Su tercera obra feminista De l'excellence des hommes contre l'égalité des sexes es editada en París en 1675, 1679, 1690 y 1692, es una «autorrefutación». En ella Poullain intenta desmontar, uno a uno, los argumentos en contra de la igualdad de las mujeres, refutándose a sí mismo y reconstruyendo argumentos basados en la razón. Amorós señala que en esta obra encontramos una crítica, muy adelantada para su tiempo, de la valoración positiva del progreso científico-técnico, y una «conjetura histórica sobre el estado de naturaleza que contiene la idea cartesiana del «bon sens» de carácter universal, previendo el peligro que podía presuponer la corrupción del desarrollo técnico no-racional vencido por la razón práctica kantiana "avant la lettre»»"

\section{Influencia social de los principios cartesianos}

Henri Pieron en su artículo publicado en 1902 «De l'influence sociale des principes cartésiens. Un précurseur inconnu du féminisme et de la Révolution: Poullain de la Barre» rescata del olvido la figura de ese "precursor desconocido» del feminismo y de la Revolución, que logró aplicar los principios de la duda metódica y el libre examen racional a las cuestiones del ámbito social, desarrollando ideas con y «contra» Descartes; es decir aplicándole el racionalismo al mismo cartesianismo ${ }^{17}$. Para Pieron el espíritu ilustrado de nuestro autor, las consecuencias revolucionarias de su pensamiento y de sus reflexiones, van en consonancia

${ }^{14}$ L'Éducation, p. 267 y p. 328 y ss. En la traducción al castellano citada, p. 188.

${ }^{15}$ Idem p. 325 original. Traducción propia.

${ }^{16}$ Amorós, (1995) op. cit., p. 116.

${ }^{17}$ Publicado en Revue de Synthèse Historique, V, pp. 153-85, 270-82, ver p. 153. 
con su igualitarismo feminista, un pensamiento feminista «verdaderamente original y radical en sus planteamientos» ${ }^{18} \mathrm{y}$ por ello compara su pensamiento con el de Pascal, La Fontaine; La Bruyère, entre otros.

Descartes trazó una nueva categorización de la realidad a partir de la crítica a los saberes medievales y renacentistas. El racionalismo cartesiano se propone una verdadera reforma del conocimiento a partir de la plena confianza en la razón como única fuente válida para el conocimiento, comprensión o interpretación verdadera de la realidad, la universalización del bon sens, y la aplicación de un método sencillo y claro que nos permita conocer. En la antropología cartesiana, el ser humano es un ser que se ha vuelto sobre sí mismo y que sólo puede conocer directamente su propio pensamiento; las cosas se conocen mediante ideas, por ello era posible dudar de su existencia y la realidad del mundo ya no era evidente: había que deducirla. Este giro antropocéntrico asegura la universalidad del «bon sens» y construye una concepción moderna de la subjetividad ${ }^{19}$.

Todo ello se traduce en un movimiento criticista contra la autoridad y en favor de la autonomía intelectual. Descartes, con su revolucionaria imagen de la libertad humana, abre una perspectiva científica y también moral que precisa un nuevo sujeto autorresponsable, que ya no encaja con los viejos esquemas. El ideal es que cada uno, con la luz de su razón, pueda encontrar el camino al margen de dogmas y tutelas.

Ahora bien, como señala Amorós ${ }^{20}$, en este «nuevo sujeto» cartesiano se unen, por un lado la necesidad de un público con capacidad autónoma de juzgar e intersubjetivizar, y por otro lado ese "público» burgués habermasiano ${ }^{21}$ que resulta de la continúa erosión de la estratificación estamental de la sociedad; dando forma a ese nuevo "público" emergente que es universal, al cual se dirige el Discurso del Método y que se basa en la unidad de la razón para toda la especie. Todos los sujetos tienen capacidad autónoma de juzgar — por el bon sens - e irracionalizar los prejuicios a la luz de la razón y pueden alcanzar el conocimiento por ellos mismos.

${ }^{18}$ Ídem, p. 153-154.

${ }^{19}$ Cfr. Turro, (1985), Descartes. Del hermetismo a la nueva ciencia, Barcelona, Anthropos.

${ }^{20}$ Cfr. Amorós, (1997), op. cit., Capítulo II.

${ }^{21}$ Habermas, (1981) Historia y crítica de la opinión pública, trad. Antoni Doménech y Rafael Grasa, Barcelona, Gustavo Gili. 
Poullain de la Barre pragmatiza el cogito cartesiano ${ }^{22}$, desarrollando la influencia social de los principios cartesianos y del proyecto racionalista. Así de la reforma de la mente, nace un proyecto ilustrado de reforma social. ${ }^{23}$ Nuestro autor no solamente amplió el programa cartesiano, incluyendo el ámbito de las costumbres «les moeurs», sino que además radicalizó el programa cartesiano de irracionalización del prejuicio haciendo explícita la participación de la mujeres en ese "público» que, en virtud de su común bon sens, tenía capacidad autónoma de juzgar e intersubjetivizar. Su programa empieza por irracionalizar el más tenaz de los prejuicios: la desigualdad de ambos sexos ${ }^{24}$.

El interés de Poulain por la acción le lleva a buscar una inflexión ético-politica del cartesianismo, formulando el principio de libertad personal de las mujeres que toma sentido a partir de los efectos de diferenciación social que él había constatado. En esta perspectiva, se entiende el «yo existo, porque pienso, porque actúo $»^{25}$, poniendo en primer lugar la acción, ya que la sociabilidad humana cumple una función finalista de vivir en sociedad, la de comunicar mediante el cuerpo, entendido éste como canal e instrumento de todo nuestro conocimiento y de todas nuestras acciones y, a la vez, como nexo de cohesión social de los hombres entre ellos y del hombre con la naturaleza. Para nuestro autor la educación es el fundamento de la adecuada relación intersubjetiva y con la naturaleza. Sólo mediante el desarrollo de la razón universal e igualitaria de hombres y mujeres podemos alcanzar la utopía racional.

${ }^{22}$ Cfr. Amorós, (1997), op. cit., cap. II, pp. 116-122.

${ }^{23}$ Cfr. Pieron, (1902), op., cit.

${ }^{24}$ L'Égalité, p. 140 y en L'Éducation "advertisement"

${ }^{25}$ L'Éducation p, 114.

${ }^{26}$ L'Éducation p. 332-336.

${ }^{27}$ Así pues, «la filosofía de Descartes... era una liberación — para Poullain—, ha sido la ocasión de una puesta en cuestión, no solamente de sus ideas, sino también de su existencia. Ocasión de liberarse de una escolástica engullida sensata y pasivamente y una profesión a la que se ha esposado sin vocación. Detrás de su esclavitud silenciosa soñaba un rebelde que todavía no había encontrado sus armas. Cuando las hubo descubierto, él estaba en disposición de uso que es un acto de pura libertad. El saber, el libre arbitrio, la voluntad, es lo que él retuvo del pensamiento de Descartes, Poullain - ha visto que esta filosofía, contrariamente a la otra, era una filosofía abierta, y es por lo que él abrió las puertas a aquello que Descartes había cerrado» Alcover, op., cit, p. 78-79. 
De forma sintética podemos afirmar que nuestro autor asume la filosofía y la epistemología cartesiana ${ }^{26}$ en función de sus intereses emancipatorios ${ }^{27}$, por ello se centra sobre todo en el Discurso del Método: su rechazo al escolasticismo, la práctica de la duda, la distinción entre la unión del alma y el cuerpo ${ }^{28}$ (a partir de la cual construye su argumento igualitario ${ }^{29}$ y se aleja del pensamiento de Descartes en las cuestiones metafísicas y en la decisión de no «respecter la coutume de son pays», sino que más bien todo lo contrario, decide luchar contra la costumbre y la tradición establecida ${ }^{30}$. Por otra parte, la filosofía cartesiana le es útil para afirmar que las diferencias y desigualdades son fabricadas en cada historia individual o colectiva y que estas diferencias producen la exclusión ${ }^{31}$.

\section{Las repercusiones teóricas de su pensamiento}

En la historia de la filosofía es necesario situarle en tres niveles: como precursor del feminismo; como influencia soslayada en otros grandes pensadores como Montesquieu y Rousseau, principalmente; y finalmente como signo adelantado de las Luces y de la Revolución

\section{Como precursor del feminismo}

Amorós señala que Poullain de la Barre es una de esas «piezas del puzzle» que configuran el proyecto ilustrado que hay que rescatar del olvido en la elaboración de la memoria crítica del feminismo. Si bien nuestro autor no es el primero en hablar de la igualdad sexual, ya que a partir del Renacimiento la cuestión ya era planteada por ejemplo en Christine de Pisan y Marie de Gournay entre otras. Además la cuestión del saber y del poder de las mujeres estaba a la orden del día en el siglo XVII—-tanto por las Frondeuses como por las Précieuses-. Pero Poulain establece, dentro de ese campo tan rico en publicaciones y controver-

${ }^{28}$ L'Excellence, p. 10 y pp. 109-111. Como afirma Fraisse, «separando el espíritu y el cuerpo, Descartes y su filosofía barren brutalmente todos los argumentos contra la igualdad de las mujeres, los argumentos fundados en general sobre la particularidad de su cuerpo» op. cit., p. 32.

${ }^{29}$ L'Excellence, cap. III, pp. 155 y ss.

${ }^{30}$ L'Éducation, pp. 324-325.

${ }^{31}$ Cfr. Fraisse, G. op. cit, p. 28. 
$\operatorname{sias}^{32}$ una verdadera ruptura teórica: él fundamenta su análisis de la igualdad de los sexos en el concepto cartesiano de que la mente no tiene sexo, por tanto hombres y mujeres pueden acceder al conocimiento en la misma medida.

Una vez reconocida la igualdad del espíritu y de cerebro entre los sexos, él va a explicitar las condiciones de esta igualdad para todos sobre la base de la universalidad de la razón, tomando el más antiguo de los prejuicios, la relación entre los sexos, como ámbito por excelencia de la irracionalidad y como punto de par$\operatorname{tida}^{33}$. En Poulain el prejuicio es una cuestión filosófica, un problema filosófico en sí mismo. Geneviève Fraisse afirma que, mientras en Descartes el prejuicio es un problema real, un punto de partida teorica, en Poulain se denuncia ese prejuicio, pero sobre todo se reflexiona sobre la historicidad del mismo y la forma de combatirlo ${ }^{34}$. Entonces, nuestro autor, comienza por irracionalizar el prejuicio de la desigualdad sexual, y cumpliendo con su temprana vocación filosóficocrítica elabora una crítica contra el poder y el dominio masculino que va más allá y se convierte en un ataque contra el abuso de poder en las estructuras sociales. Podemos decir que elabora un verdadero «ataque epistemológico» contra la autoridad tradicional en todos los campos de la actividad humana e incluso pudiendo llegar, en mi opinión, a formular un primer concepto tentativo de cambio social. Así, podemos ver en su obra el primer intento de construir un igualitarismo universal societario que podría llamarse «cartesianismo social», como anticipada expresión de la Modernidad.

En síntesis, la igualdad que propone debe ser absoluta y esta afirmación se inscribe en dos niveles determinantes: la igualdad de saber y la igualdad de poder. Las mujeres tienen el derecho de cultivar su espíritu en todas las áreas del conocimiento y el derecho a utilizar su saber en todas las funciones sociales comprendiendo el gobierno, la guerra, la iglesia, etc. Autores como Pieron o Gappin ${ }^{35}$, están prácti-

${ }^{32}$ Ascoli, en la obra ya citada, toma y compara la larga lista dada por Albistur y resitúa estas numerosas apologías comparándola con otra producción literaria popular de la época sobre las mujeres que es la misógina y que está reagrupada bajo el título de «Miroir des femmes». Ver a estos propósitos el análisis d'Arlette Farge: Le Miroir des femmes, ed Montalba, 1982, cit. por Fraisse, op., cit., p. 28. Ver además Albistur M. et Armogathe, D. (1977) L. 'Histoire du féminisme farçais du Moyen-âge à nos jours, Paris, Ed. Des Femmes.

${ }^{33}$ L'Égalité, pp. 4-5 - 100-101.

${ }^{34}$ Cfr. Fraisse, op. cit., p. 39-40.

${ }^{35}$ Op., cit. 
camente de acuerdo en defender la causa de la igualdad de saber, sin embargo, son más reacios a aceptar la igualdad de poder o a abrir el espacio público a la mujer, y en su época, dos siglos más tarde, les sigue pareciendo excesivo el pensamiento de Poulain. Sus tesis igualitaristas en base a las cuales propone un programa educativo para mujeres y hombres se adelantan en más de un siglo a las tesis defendidas por Fénelon y Mme de Maintenon, son mucho más radicales, y en consecuencia más adecuadas a la problemática de principios del siglo XX; así pues es solamente a nivel de las instituciones y de las leyes que las tesis de Poullain sobre las mujeres se pueden traducir en actos y para ello deberíamos esperar a los primeros reconocimientos de derechos en el siglo XIX ${ }^{36}$.

\section{Influencia soslayada en otros grandes pensadores como Montesquieu y Rousseau, principalmente}

Poullain ha prefigurado el relativismo histórico y geográfico de la teoría de los climas de Montesquieu; ha esbozado una conjetura histórica sobre el estado de naturaleza. El texto tiene grandes similitudes con el desarrollado luego por Rousseau. No hay suficientes pruebas documentales sobre la probabilidad de que los libros de Poullain hayan estado en posesión de los dos filósofos mencionados. En el caso de Rousseau, que fue secretario de Mme Dupin quien tenía en su biblioteca una copia de L'Égalité, es muy probable que éste accediera a esa .obra. Son más que evidentes las similitudes en algunos pasajes. Amorós afirma que «El libro V de El Emilio de Rousseau, «La educación de Sofía», es escrito contra Poulain de la Barre, su referente polémico silenciado, y no refleja sin más su época, ya que cuando afirma que «la subordinación de las mujeres no se basa en prejuicios sino en la razón» está contradiciendo, diciendo-en-contra de aquel que afirmaba en De L'égalité: «La desigualdad de los sexos no está basada en la razón, sino en el prejuicio» ${ }^{37}$.

Bernard Magnè lanza la hipótesis de que el "philosophe tres galant» de las Lettre persane, XXXVIII, no es otro que el mismo Poullain «Las semejanzas entre los

${ }^{36}$ Cfr. Fraisse, op. cit., p. 28- 30.

${ }^{37}$ Amorós, (2008) Mujeres e imaginarios de la globalización: reflexiones para una agenda teórica global del feminismo, Argentina, Homo Sapiens Ed. p. 123. 
dos textos son precisas y numerosas, por lo que no parecen ser fortuitas..., sin embargo podemos aportar una prueba decisiva: nosotros sabemos que efectivamente Montesquieu leyó L'Egalité des deux sexes. (...) en la Bibliothéque Nationale hay un ejemplar del mismo con una nota marginal y manuscrita... $»^{38}$.

Otros autores insisten en la menos conocida parentela de los textos de Poullain y el «connais-toi toi-même» de Montaigne, viendo así esquematizarse el concepto de individuo en toda su novedosa acepción, evidentemente propicia para la mujer ${ }^{39}$.

\section{Signo adelantado de las Luces y de la Revolución}

Tanto Celia Amorós como Siep Stuurman ${ }^{40}$ insertan el pensamiento de nuestro autor en el contexto más amplio de la Ilustración. Hazard en su clásica obra La crise de la conscience européenne, argumentó que las ideas que dieron origen a la Ilustración francesa del siglo XVIII ya habían sido expresadas en 1680, como ya hemos señalado, aunque nuestro filósofo está totalmente ausente del pensamiento de Hazard, su trayectoria está inserta en ese contexto y tanto su itinerario personal como intelectual nos hacen ver que nuestro autor pertenece por derecho propio a esa nueva generación de intelectuales que habiendo abandonado la «cautela» de Descartes en asuntos religiosos y políticos, se lanzaron a aplicar la crítica racionalista a todos los ámbitos intelectuales y sociales, desde el derecho y la ciencia, la filosofía y la moral hasta la teoría política, desmoronando los fundamentos del pensamiento tradicional.

Stuurman presenta varios argumentos para incluir a nuestro autor en ese canon de ilustres precursores de la Ilustración, entre ellos: la intercepción entre "feminismo e Ilustración», unido a su igualitarismo inclusivo, que no sólo crítica la supremacía masculina y la jerarquización social, sino que además aporta una conjetura histórica del origen de propiedad y el poder, enfatizando la igualdad entre naciones y etnias. En esta línea, Amorós y Fraisse definen el pensamiento y la obra del filósofo cartesiano como momento clave en la articulación teórica

${ }^{38}$ Magnè, (1968): «Une source de la Lettre persane XXXVIII? 'L'Egalité des deux sexes' de Poullain de la Barre» Revue d'histoire littéraire de la France, mai-aout, pp. 407-414.

${ }^{39}$ Christine Fauré, desarrolla está idea en La Démocratie sans les femmes, Essai sur le libéralisme en France, Puf, 1985, p. 128. 
del feminismo moderno, como la senda perdida o el eslabón lógico entre el pensamiento de Descartes y Rousseau ${ }^{41}$. Fraisse señala que la filosofía igualitaria de Poulain es realmente la primera tentativa sostenida de aplicar el razonamiento cartesiano al análisis de la sociedad, de la autoridad, y el poder. Además con De l'égalité estaríamos asistiendo a un verdadero cambio en el estatuto epistemológico de la controversia o "guerra entre los sexos», pues «la comparación entre el hombre y la mujer abandona el centro del debate, y se hace posible una reflexión sobre la igualdad $»^{42}$, siendo el primer pensador en la Europa moderna que construye toda su filosofía social sobre el concepto universalista de igualdad.

El segundo argumento de Stuurman es la condición de racionalista-crítico en su quehacer filosófico, signo de identidad más propio de los filósofos ilustrados del siglo XVIII, otro de los aspectos de su filosofía que ha recibido poco aten$\operatorname{dido}^{43}$.

Lo que hizo absolutamente revolucionario el pensamiento de Poullain, fue que sistematizó, bajo el marco teórico del racionalismo, una serie de discursos dispersos, elaborando de esta forma un argumento teórico coherente. Fue un feminista que se adhirió, sin reservas, a los «modernos» y fue asimismo el primer moderno que intentó integrar el feminismo dentro de una filosofía social sistemática. Como bien lo señala Stuurman, esta combinación puede parecer lógica vista desde nuestros tiempos, pero aplicando la retrospectiva histórica vemos que su posición teórica en 1670 contenía los temas claves que serían desarrollados en la Ilustración y articulan un discurso claramente revolucionario.

Finalmente, queremos subrayar otro pilar de su pensamiento, como es la visión crítica de la historia como mantenedora y legitimadora de los prejuicios y la dominación. Poullain señala la existencia de unas estructuras sociales profundas - he aquí otro punto de vista novedoso para su tiempo- que es necesario visibilizar: «Lo que hace la unidad de la historia, hasta ahora, es el hecho

\footnotetext{
${ }^{40}$ Stuurman, (2004), op., cit.

${ }^{41}$ Cfr. Cobo, (1995) Fundamentos del patriarcado moderno. Jean Jaques Rousseau, Madrid, ed. Cátedra.

${ }^{42}$ Fraisse, op cit., pp. 27-41. Traducción propia.

43 Stuurman, (1997) «Social Cartesianismo. François Poulain de la Barre and the Origins of the Enlightenment» en Journal of the History of Ideas, Vol. 58, No. 4, pp. 617-640. Ver pp. 618

${ }^{44}$ L'Egalité, op. cit. p. 15.
} 
de que siempre la fuerza prevaleció sobre la razón» ${ }^{44}$ Bernard Magnè, en su tesis inédita ${ }^{45}$ sobre nuestro autor, señala el germen del materialismo y del estructuralismo latente en su pensamiento. Seguramente podemos considerarle como un precursor de la sociología antes que Comte. Esta reconstrucción de la historia como descubrimiento de las estructuras de poder latentes de la realidad social interesa a la teoría feminista para rastrear esta idea del dominio de la fuerza, entendiéndola desde la construcción del patriarcado como institución ${ }^{46}$.

En suma, podemos concluir que nuestro autor puede considerarse una avanzadilla del pensamiento crítico ilustrado que reivindica la igualdad como una idea fuerza para hombres y mujeres mucho antes de la Revolución.

\section{Bibliografía}

\section{Obras de Poulain}

(1672). Les rapports de la langue latine avec la françoise, pour traduire élégamment et sans peine avec un recueil étymologique et méthodique de cinq mille mots franeois tirez immédiatement du Paris, Veuve Claude Thiboust et Pierre Esclassan.

(1673). De l'égalité des deux sexes; discours physique et moral oú l'on volt l'importance de se défaire des préjugez,- Paris, chez Jean du Puis.

(1674). De l'éducation des dames pour la conduite de l'esprit dans les sciences et dans les moeurs, entretins Paris. chez Jean du Puis.

(1675). De l'excellence des hommes contre l'égalité des sexes . - Paris, chez Jean du Puis.

(1720). La doctrine des Protestans sur la liberté de lire l'ecriture Sainte, le Service Divin en langue entendue, l'Invocation des Saints, le Saerement de I'Eucharistie, justifiée par le Missel Romain et par des Reflexions sur chaque point. Avec un commentaire philosophique sur ces paroles de Jésus-Christ : "Ceci est mon Corps, Ceei est mon Sang». - Mathieu. chap. XXVI V. 26». Genève.

(1691). Essai des remarques particulières sur la langue françoise pour la ville de Genève, rédigé par Poulain de la Barre, (desconocemos su editorial).

\footnotetext{
${ }^{45}$ Magnè, (1964) op. cit.

${ }^{46}$ Amorós, (1985), Hacia una crítica de la razón patriarcal, Barcelona, Anthropos.
} 


\section{Sobre el autor}

Amorós, C., coord. (1992). Actas del Seminario Permanente Feminismo e Ilustración 19881992, Instituto de Investigaciones Feministas de la Universidad Complutense de Madrid y Dirección General de la Mujer de la CAM, Madrid.

- (1995). Tiempo de feminismo. Sobre feminismo, proyecto ilustrado y postmodernidad, Madrid, Cátedra, Colección Feminismos.

Amorós, C. y Cobo, R. (2005). «Feminismo e Ilustración» en Celia Amorós y Ana de Miguel (eds.), Teoría feminista: de la Ilustración a la globalización, vol. I, Madrid, Minerva Ediciones Biblioteca Nueva.

Abensour, L. (1923). La Femme et le Féminisme avant la Révolution. Paris, Leroux.

Alcover, M. (1978). «The Indecency of Knowledge», Rice University Studies, vol. 64, no 1 , Winter, pp. 25-39.

Ascoli, G. (1906). «Essai sur l'histoire des idées féministes en France du XVIéme siècle à la Révolution», Revue de synthèse historique, XIII, pp. 25-57.

BAYLE, P. (1730). Dictionnaire historique et critique, 4éme éd. Amsterdam, Brunei.

Faure, C. (1985). «Poullain de la Barre, sociologue et libre penseur », Corpus nº 1, pp. 43-51.

Fraisse, G. (1985). «Poullain de la Barre, ou le procès des préjugés », Corpus n 1, pp. $27-41$.

Grappin, H. (1913). "Notes sur un féministe oublié, le cartésien Poulain de la Barre», Revue d'histoire littéraire de la France, pp. 852-867.

Magne, B. (1964). Le Féminisme de Poulain de la Barre, origine et signification, thèse dactylographiée pour le doctorat de 3ème cycle. Université de Toulouse.

MORERI (1733). Le Brand dictionnaire historique, ou le mélange curieux de l'histoire sacrée et profane. Basle (vol. 6, Additions).

Pieron, H. (1902). "De l'influence sociale des principes cartésiens. Un précurseur inconnu du féminisme et de la Révolution, Poulain de la Barre», Revue de synthèse historique (2ème sem.), pp. 153-185 et 270-282.

ReYNier, G. (1933). La Femme au XVIIème siècle. Ses ennemis et ses défenseurs. Paris, Plan.

StuUrman, S. (1997). «Social Cartesianism: François Poulain de la Barre and the origins of the enlightenment", Journal of the history of ideas, vol. 58, no 4, pp. 617640. 
- (2004). François Poulain de la Barre and the Invention of Modern Equality, Cambridge (Mass.), Harvard University Press.

Recibido: 22/02/2011

Aceptado: 4/04/2011 